\title{
Molecular Orientation of Individual LCP Particles in Injection-Moulded PPS/LCP Blends
}

\author{
H.-J. Kestenbach ${ }^{\mathrm{a} *}$, K.-D. Rogausch ${ }^{\mathrm{b}}$ \\ ${ }^{a}$ Department of Materials Engineering, Universidade Federal de São Carlos, \\ 13565-905 São Carlos - SP, Brazil \\ ${ }^{\mathrm{b}}$ Institute of Materials Science, Universität Dortmund, 44221 Dortmund,Germany
}

Received: April 26, 2002; Revised: December 12, 2002

\begin{abstract}
Polarized light microscopy was used to investigate the presence of preferred molecular orientation in the LCP phase of PPS/LCP blends after injection moulding. Normal birefringence effects appeared to be complicated by artifacts due to sample preparation and by the complex nature of polarized light transmission through a multicomponent sample. It was found, however, that, during low-temperature cutting of optically transparent thin sections on a standard microtome, individual LCP particles could be separated from the PPS matrix, and their birefringence analyzed separately. Preferred orientation was detected only in LCP fibrils which dominated in skin regions, but not in droplet-shaped particles which had formed in core regions. Quantitative measurements indicated that the molecular orientation of the fibrils increased linearly with their lengthto-diameter aspect ratios which ranged from 15 to 50. Even for the highest aspect ratios, however, the degree of orientation was always less than that which could easily be introduced into pure LCP thin-film samples by manual shearing.
\end{abstract}

Keywords: polymer composites, LCP, molecular orientation, birefringence

\section{Introduction}

Liquid crystalline polymers (LCP) are becoming serious candidates as a reinforcing phase in thermoplastic matrix polymer composites ${ }^{1}$. Originally, the objective was the fabrication of so-called molecular composites ${ }^{2}$ in which the proper rigid-chain segments of the LCP molecule would form the structural reinforcing elements on an ultra-fine scale. However, miscibility problems with thermotropic as well as lyotropic LCP's have prevented the formation of such a fine distribution on a molecular scale ${ }^{3,4}$. More "downto-earth" objectives of current research and development proposals try to take advantage of another specific property of LCP materials which is the easy molecular alignment of the LCP molecules in its mesophase ${ }^{5}$. Thus, composites may be formed in which the reinforcing phase forms highstrength fibrils which confer increased strength to the composite in the direction of preferred alignment, in a similar way as other reinforcement materials such as glas or aramid fibres $\mathrm{do}^{6-9}$. In the case of LCP fibres, however, the rein- forcing material is in its liquid (mesophase) state during blend processing ${ }^{10}$ (for example, extruded or injectionmoulded products), with important benefits to moulding capacity and tool life during the production cycle ${ }^{11}$.

There are several factors which determine the efficiency of a reinforcement phase in directional composites ${ }^{12}$ : First, the longitudinal aspect ratio (length-to-width ratio of the reinforcing particles). Second, the interfacial adhesive strength between the reinforcing phase and the matrix. Third, the proper strength of the reinforcing particles in the direction of preferred alignment. In case of a polymer phase forming the reinforcement (for example the case of LCP reinforcing particles), particle strength will be a funcion of molecular alignment ${ }^{13}$. It is for this reason that the molecular orientation within LCP particles of a thermoplastic matrix polymer blend adquires technological importance, and that the present research objective was aimed at studying the molecular orientation of individual particles by a new experimental technique.

Injection-moulded samples of poly(p-phenylene sul-

*e-mail:dhjk@ power.ufscar.br 
phide) PPS/LCP composites were investigated in the present work. X-ray diffraction or infrared vibrational spectroscopy ${ }^{14}$ are usually employed to determine the molecular orientation in polymers. Both techniques give average results and do not detect local variations in orientation, a particular handicap in the case of injection-moulded parts which tend to show a strong skin-core effect ${ }^{15}$. For example, the second-phase LCP particles in thermoplastic matrix polymer composites may form fibrils close to the surface and droplets in the central zone of injection-moulded specimens ${ }^{16,17}$. A light-optical method based upon the birefringence of oriented polymers was therefore used in order to investigate the degree of molecular orientation in the LCP phase as a function of particle geometry.

\section{Experimental Procedures}

Blends of PPS-Fortron (a Hoechst-Celanese product) with Vectra A-950 (a wholly aromatic liquid crystalline copolyester also from Hoechst-Celanese) were prepared by mixing in a Werner Pfleiderer ZSK-30 co-rotating twin screw extruder, using a temperature profile of $250 / 270 / 280 / 285 / 290 / 300{ }^{\circ} \mathrm{C}$ (melting temperatures of the starting materials were $280{ }^{\circ} \mathrm{C}$ for PPS and about $300{ }^{\circ} \mathrm{C}$ for the LCP), $150 \mathrm{rpm}$ rotation speed, and three different PPS/LCP proportions: 95/05, 70/30, and 50/50. After pelletizing and drying under vacuum at $140{ }^{\circ} \mathrm{C}$ for $3 \mathrm{~h}$, flat sample tensile specimens were injection moulded in an automatic Arburg Allrounder 270 V/300-120 machine, using a temperature profile of $270 / 280 / 285 / 290 / 300{ }^{\circ} \mathrm{C}$, an injection pressure of $650 \mathrm{bar}$, and a mould temperature of $140{ }^{\circ} \mathrm{C}$. Overall geometry and specific dimensions of the tensile specimens are shown in Fig. 1a. Optical microscopy of thin microtomed sections and scanning electron microscopy (SEM) of liquid nitrogen fracture surfaces were used to study the shape and the distribution of LCP particles as a function of position. Size, orientation and approximate position of the microtomed sections are indicated in Fig. 1b. Molecular orientations were investigated under polarized light, using a Leica optical microscope with stress-free objectives and crossed polars.

Microtome cutting under liquid nitrogen was used to separate individual LCP particles from the injectionmoulded PPS/LCP composites. Due to the brittleness of the PPS matrix, LCP particles were freed and "jumped" during low-temperature cutting on the microtome. As explained in more detail below, these particles could be examined one by one under polarized light, and their birefringence and therefore their degree of preferred macromolecular orientation measured by a standard compensation technique. For this, a Leitz tilting compensator type B with a measuring range of $5 \lambda$ was used, installed on a Leica polarization microscope with stress-free optics.

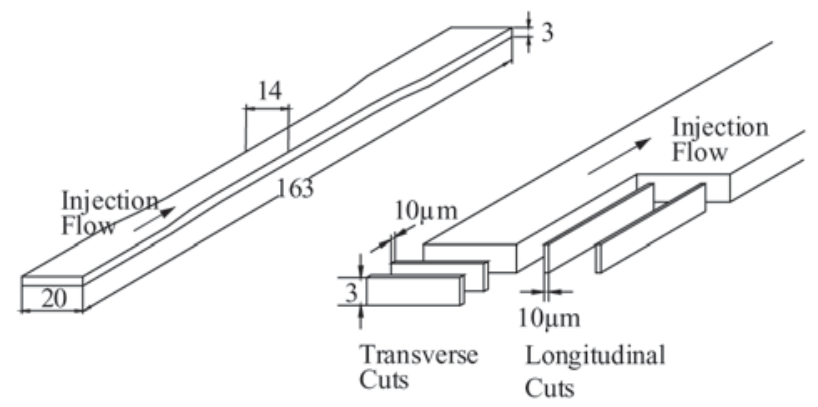

(a)

(b)

Figure 1. Specimen geometry and microtome sectioning. In a), injection-moulded tensile specimen with dimensions given in $\mathrm{mm}$. In b), orientation of thin optical microscopy sections with respect to the mould fill direction.

\section{Results and Discussion}

\subsection{Particle Morphology and Distribution after Injection Moulding}

LCP particles exhibited a typical skin-core effect after injection moulding. As shown in Fig. 2 which presents the optical microstructure in longitudinal as well as in transverse sections, elongated particles were only found in surface regions, while droplet particles (of spherical but also irregular shape) dominated the sample interior. When viewed at a higher magnification in Fig. 3, elongated particles exhibited a characteristic fibril morphology. As will be shown below, only these fibrils showed birefringence as a result of their preferred macromolecular orientation (with both macromolecules and fibrils aligned parallel to the polymer flow direction), whereas droplet particles did not exhibit any birefringence.

For both fibrils and droplet particles, the actual blend composition changed the average particle size (larger particles for higher LCP fractions) but not the particle distribution. The following investigations were therefore restricted to the 70/30 PPS/LCP composition only.

\subsection{Birefringence in Blend Samples}

At low magnifications, the entire sub-surface regions containing elongated particles presented very strong effects of birefringence. An example is shown by the colour micrographs of Fig. 4 which compares the $45^{\circ}$ intensity reinforcement with the zero or $90^{\circ}$ extinction position. When observed in longitudinal sections, Figs. $4 \mathrm{a}$ and $4 \mathrm{~b}$, this effect of birefringence corresponds to expectations and would normally be associated with the presence of preferred macromolecular alignment in the longitudinal direction. However, as shown in Figs. 4c and 4d, a similar effect of 


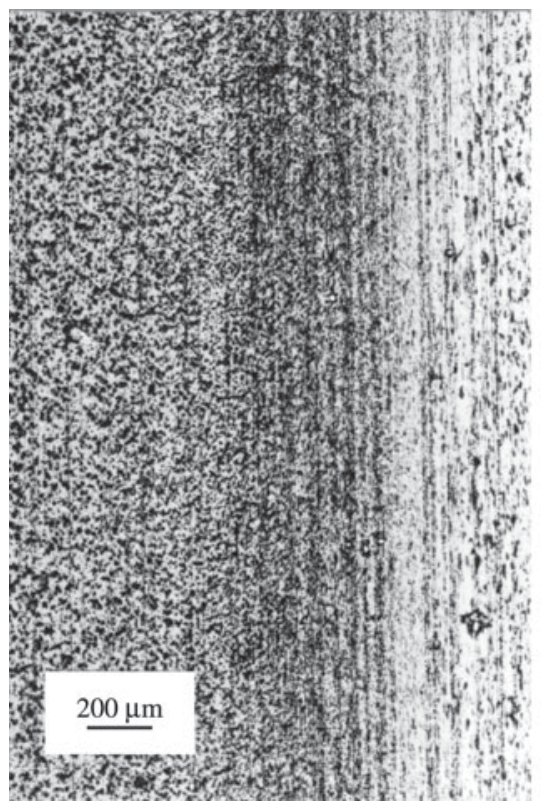

a)

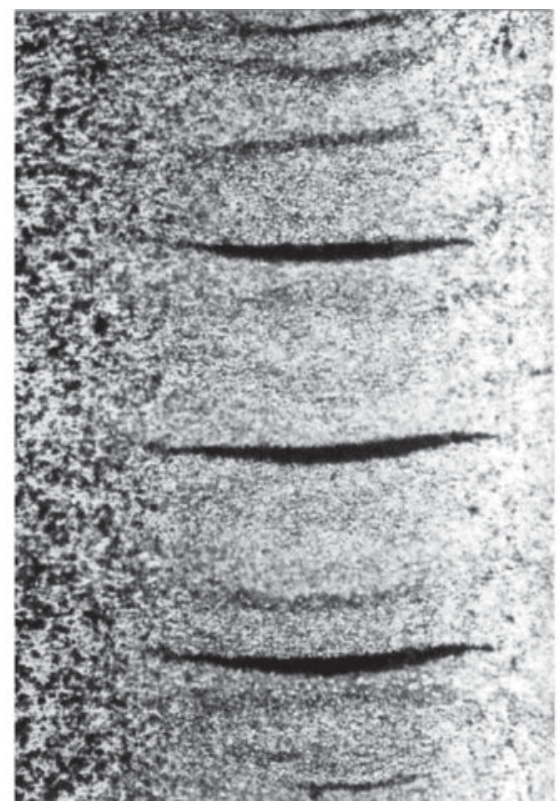

b)

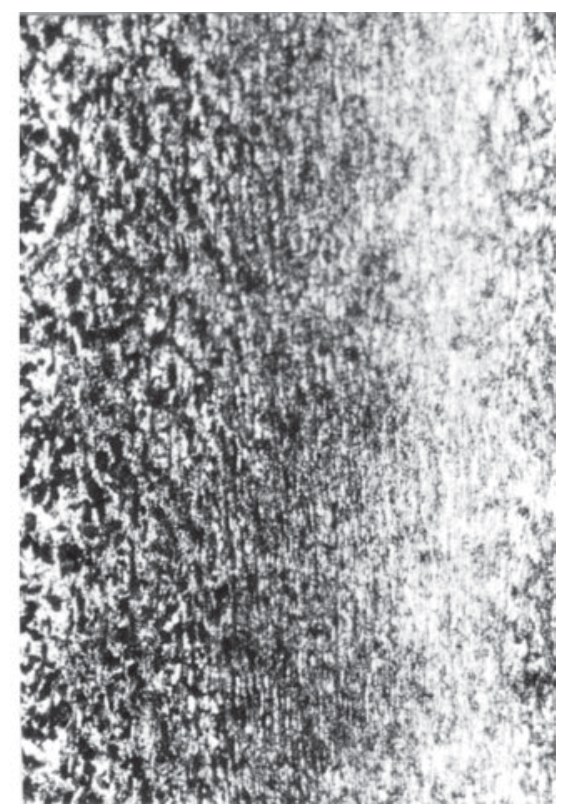

c)

Figure 2. Distribution of LCP particles after injection moulding, showing typical "skin-core" effects (surface layers at right-hand side of micrographs). PPS/LCP blend compositions of 95/05 in a), 70/30 in b), 50/50 in c). Longitudinal sections in a) and c), transverse section in b). Magnification 30x.

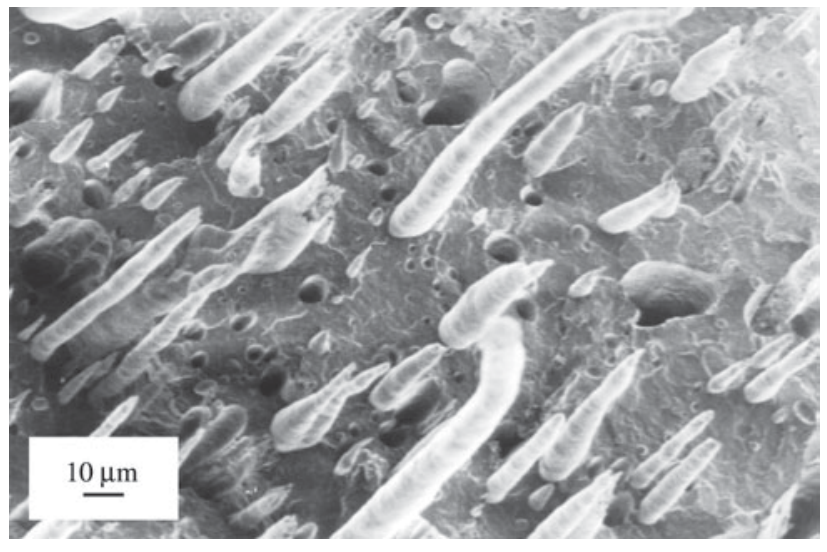

Figure 3. Scanning electron micrograph of elongated LCP particles on transverse freeze fracture surface of a 70/30 PPS/LCP blend. Magnification 500x.

birefringence was also found in transverse samples. In this case, the observed birefringence cannot be attributed to molecular alignment since the polymer flow direction was perpendicular to the transverse sample plane. Thus, part of the low-magnification birefringence effects, and certainly that part which appears in transverse sections, must come from sample preparation artifacts introduced by microtome cutting.

A totally different situation was encountered at higher magnifications for which the elongated LCP particles were resolved. As shown by the colour micrographs in Fig. 5, birefringence effects were only observed for the PPS matrix, with colour changes from yellow in the first quadrant to blue in the second quadrant. Such birefringence effects are well known to occur in a microstructure of small unresolved spherulites ${ }^{18}$ which would correspond to the expected matrix microstructure. Concentrating on the appearance of the LCP fibrils, however, no intensity or colour changes between $45^{\circ}$ reinforcement positions in Figs. $5 \mathrm{a}$ and $5 \mathrm{c}$ and the extinction position in Fig. 5b could be detected, probably due to rather complex physics which will control the transmission of light in a polymer blend sample. This point will be taken up again in the discussion.

\subsection{Particle Separation Technique}

As shown above, artifacts due to microtome cutting as well as the complex nature of light transmission through multiphase materials seemed to rule out the birefringence effect as a useful technique for studying preferred macromolecular orientation in composite samples. An alternative, of course, would be the separation of the oriented LCP particles from the disturbing spherulitic matrix. Such a separation technique was discovered almost by accident when microtome cutting was performed at liquid nitrogen temperatures. Initially, samples for optical microscopy were cut at room temperatures because of the rather high glass 


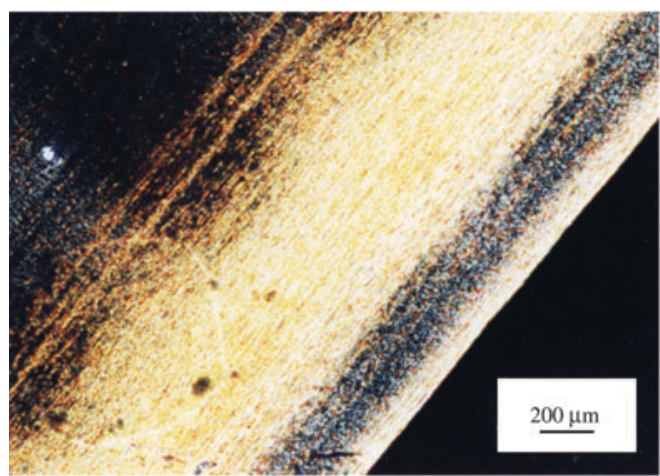

a)

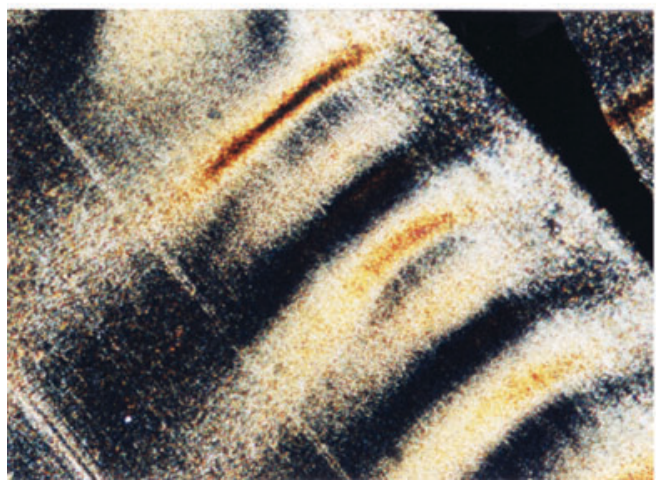

c)

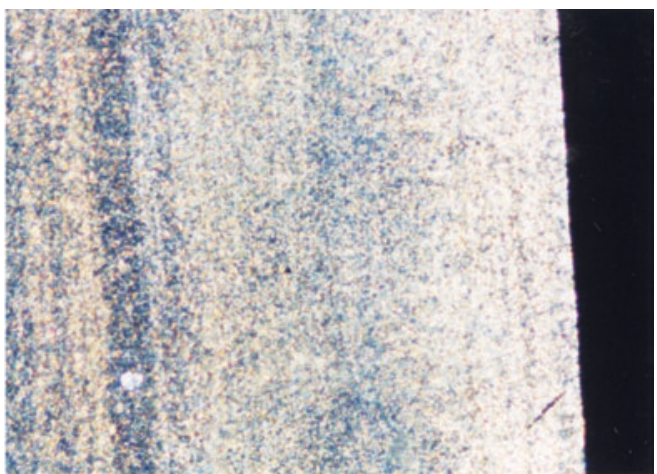

b)

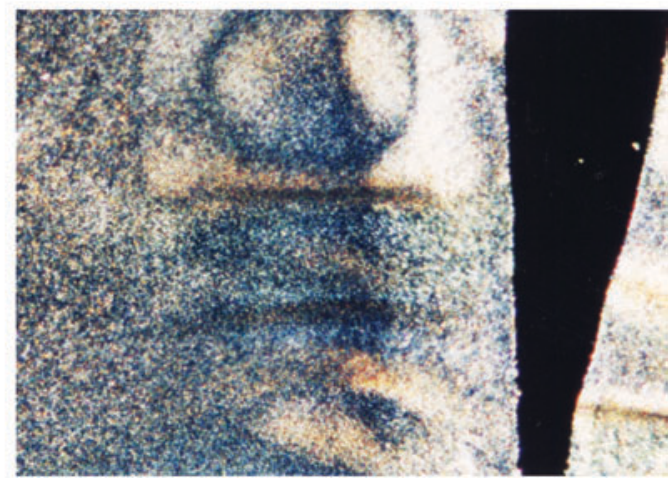

d)

Figure 4. Low-magnification birefringence effects in subsurface regions of an injection-moulded 70/30 PPS/LCP blend sample. Longitudinal section in a) and b), transverse section in c) and d). Reinforcement positions are shown at left, extinction positions are shown at right. Polarized light microscopy with $\lambda$ plate, magnification 30x.

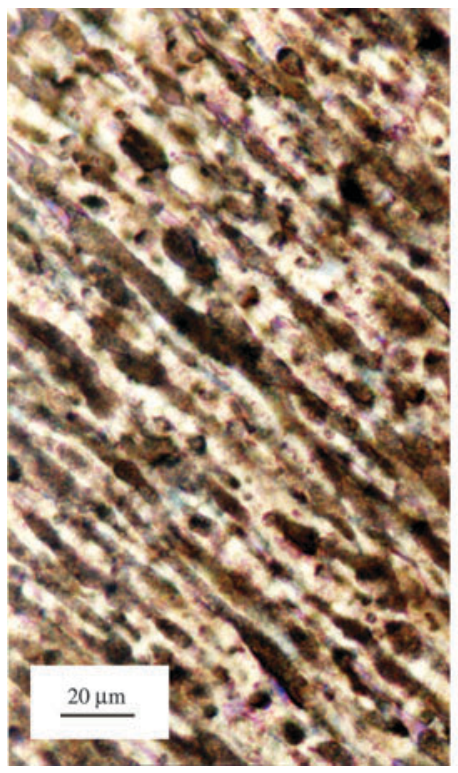

a)

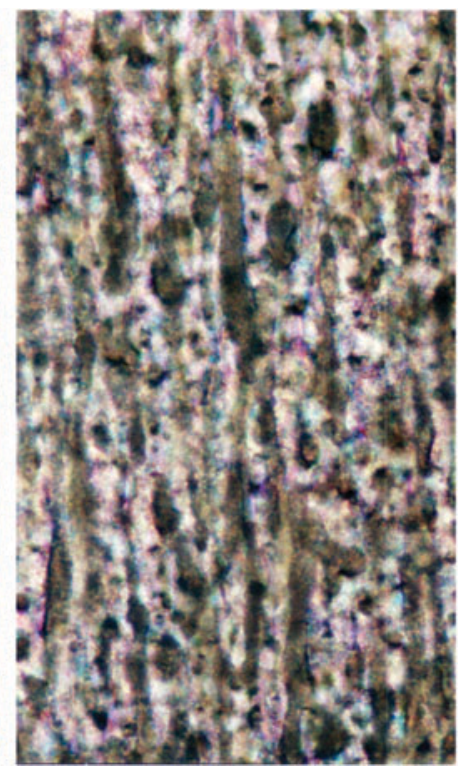

b)

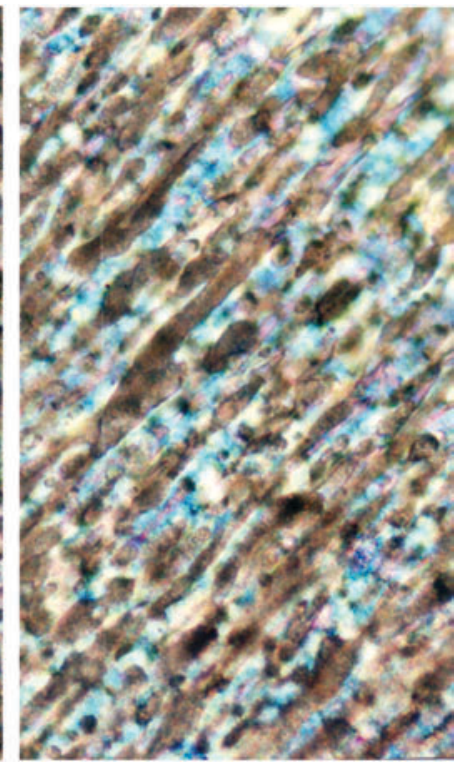

c)

Figure 5. Higher-magnification birefringence effects in subsurface region of an injection-moulded 70/30 PPS/LCP blend sample (longitudinal section). Reinforcement positions in a) and c), extinction position in b). Polarized light microscopy with $\lambda$ plate, magnification $400 x$. 
transition temperatures of both the PPS matrix ( $\mathrm{T}_{\mathrm{g}}$ between 85 and $90{ }^{\circ} \mathrm{C}$ ) and the LCP particles ( $\mathrm{T}_{\mathrm{g}}$ around $\left.140{ }^{\circ} \mathrm{C}\right)$. When the preferred orientation effects in transverse samples were noted as mentioned in section 3.2, additional cuts were performed at liquid nitrogen temperatures in order to try to avoid this artifact. Although the artifact (birefringence perpendicular to the injection flow direction) remained, the increase in sample brittleness at such low temperatures caused the PPS matrix to fracture, freeing large numbers of LCP particles. Large amounts of these particles were col-

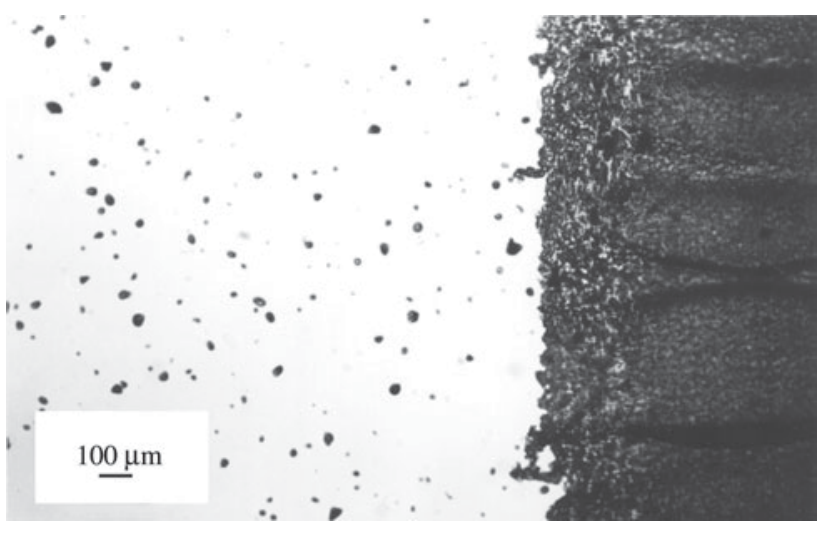

Figure 6. LCP particles separated from 70/30 PPS/LCP blend by low-temperature microtome cutting. Original transverse section at right. Optical micrograph under bright field conditions, magnification $45 x$. lected automatically by the immersion oil added after cutting in order to prepare and straigthen the thin sections between glass slides for optical microscopy observation. An example is shown in Fig. 6. Most of the isolated LCP particles were roundish in shape (droplet particles) which means that they were collected from central portions of the specimen. However, and particularly in conjunction with transverse sections, a sizable number of elongated LCP particles could be found at higher magnifications, and generally further away from the specimen edges.

\subsection{Birefringence of Isolated LCP Particles}

Some typical examples of birefringence effects which were observed for isolated particles are presented in Figs. 7, 8 and 9. Elongated fibrils were easily detected under ordinary bright-field conditions as shown in Fig. 7a. As expected, most of these fibrils, when observed under crossed polars, exhibited maximum contrast at the $45^{\circ}$ position as shown in Fig. $7 \mathrm{~b}$, and total extinction at $0^{\circ}$ or $90^{\circ}$ positions as shown in Fig. 7c.

Some of the fibrils did not lie flat on the substrate but, during sample preparation, were caught sloping up or down in the immersion oil. Such a case is shown in Fig. 8 where the birefringence effect can be seen to strongly depend on focussing: The upper left end of a sloping LCP fibril is shown focussed under bright-field conditions in Fig. 8(a). The same focussing condition was used in Fig. 8(b) where the fibril appears with its maximum contrast at the $45^{\circ}$ position. Before rotating to the extinction position, however, the lower

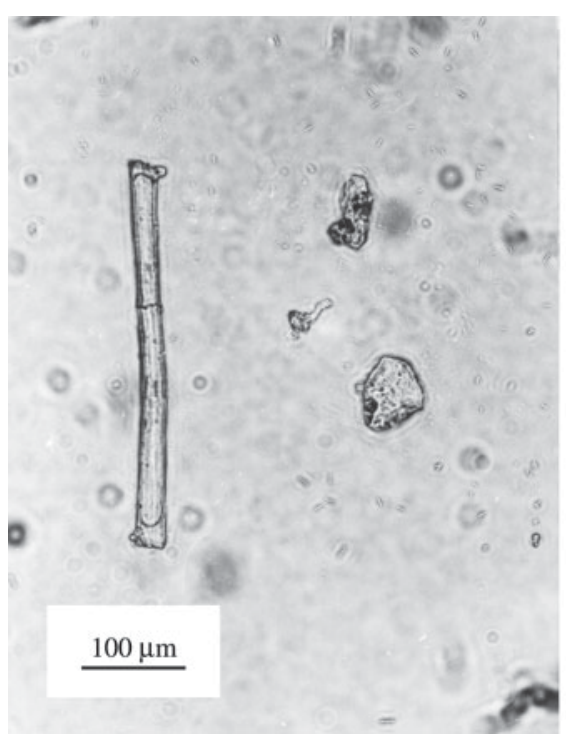

a)

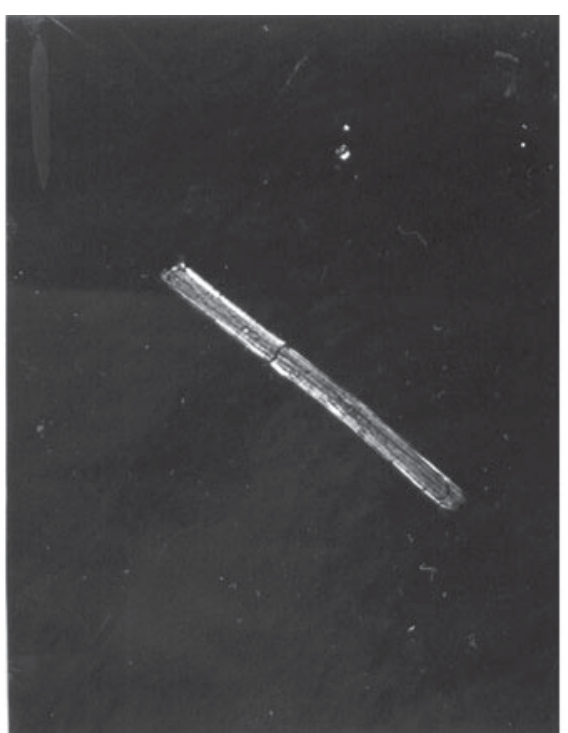

b)

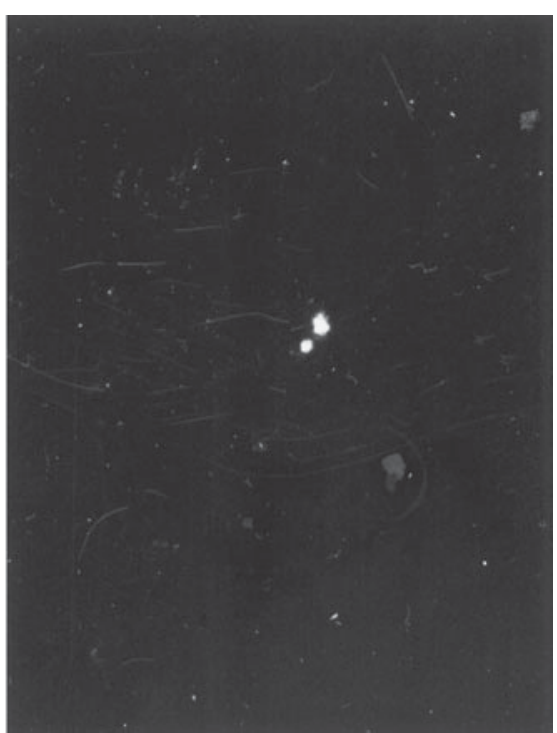

c)

Figure 7. LCP fibril showing birefringence due to preferred macromolecular orientation. Bright-field micrograph in a), polarized light with reinforcement position in b), extinction position in c). Magnification 220x. 


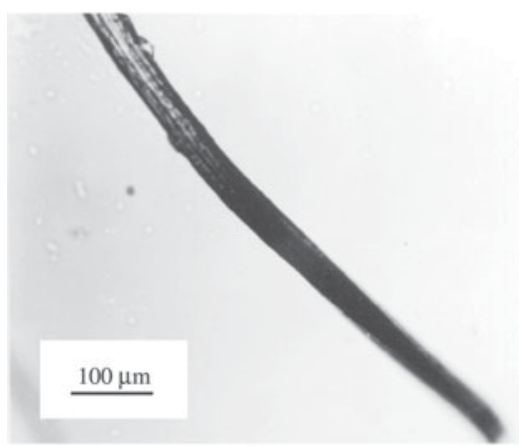

a)

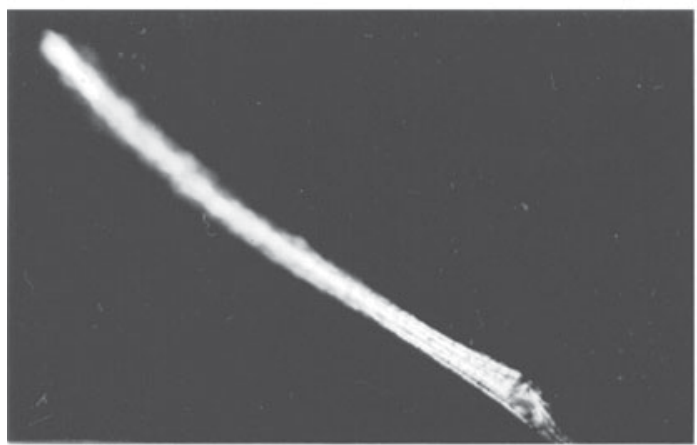

c)

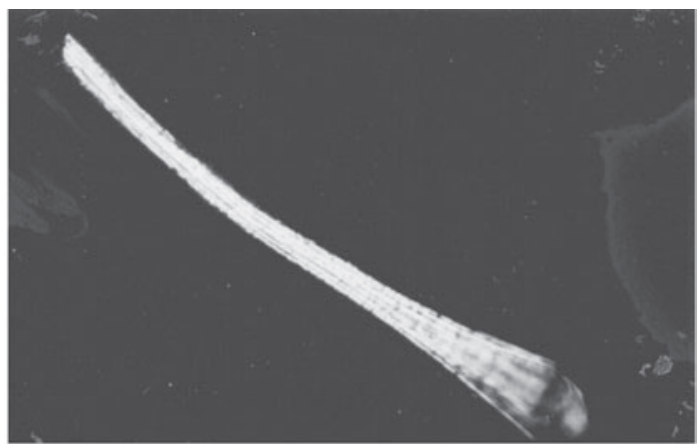

b)

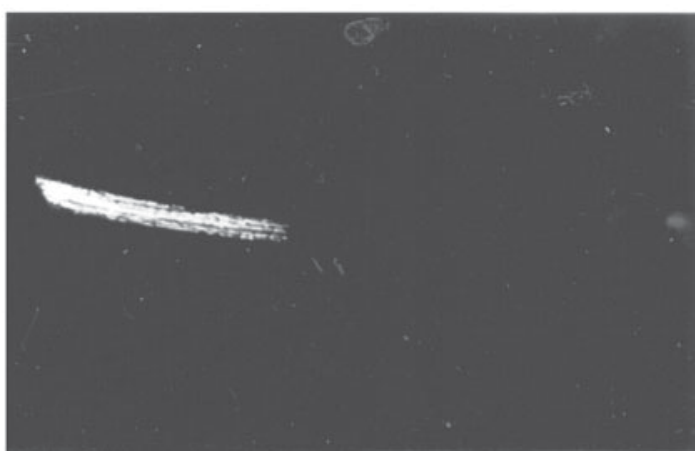

d)

Figure 8. LCP fibril showing birefringence due to preferred macromolecular orientation, but only when properly focussed. Bright-field micrograph in a), polarized light with reinforcement position in b) and c), extinction position in d). Magnification 220x.

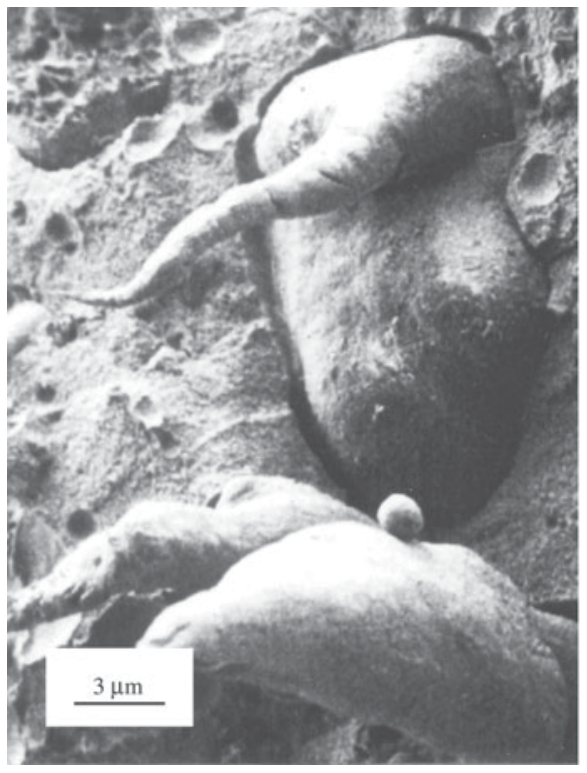

a)

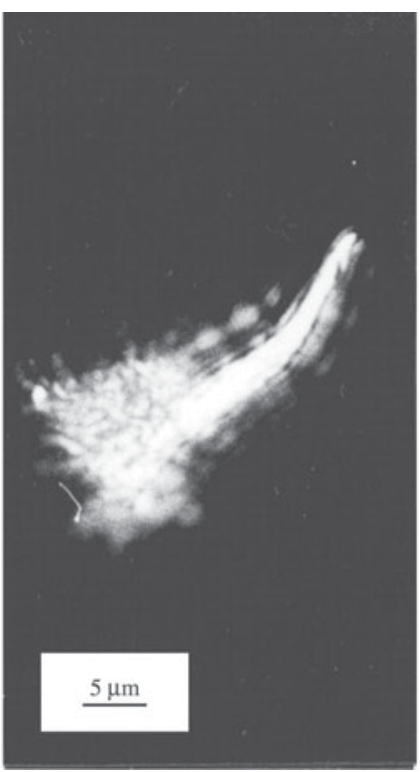

b)

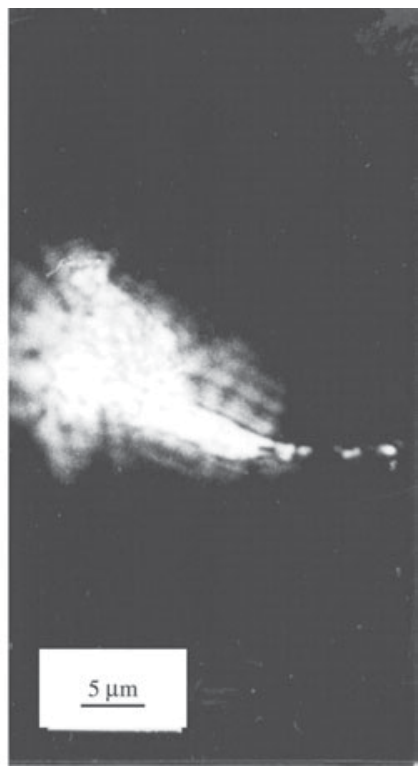

c)

Figure 9. Roundish LCP particles showing birefringence at fibrillar extremities only. Scanning electron microscopy in a), magnification 3.000x. Polarized light micrographs with reinforcement position in b), extinction position in c), magnification 1.200x. 
right end of the fibril was focussed as shown in Fig. 8c, clearly leaving its upper left end out of focus. As a result, only the right hand portion of the fibril showed extinction at the $0^{\circ}$ position as shown in Fig. 8d. Thus, a precise focussing condition is very important when the presence of birefringence is to be verified.

In general, no birefringence was found for the roundish droplet particles supposedly isolated from the interior regions of the microtome sections (see Fig. 6). However, some of these particles exhibited a hybrid morphology which can more clearly be observed in the SEM micrograph of Fig. 9a. In such cases, some birefringence was detected within the fibrillar protuberances while the main part of the particle remained without birefringence as shown in Figs. 9b and $9 \mathrm{c}$.

\subsection{Quantitative Measurements}

When a plane-polarized light beam passes through a birefringent material, it is split into two components which, travelling at different velocities, create a path difference $\Gamma$ at the sample's exit surface, responsible for interference effects and the transmittance of light in a microscope operating with crossed polars. This path difference, also known as retardation, is proportional to the difference in refractive indices for the two beam components, $\Delta \mathrm{n}$, and to the sample thickness, $\mathrm{t}$, according to

$$
\Gamma=\Delta \mathrm{nt}
$$

The magnitude of $\Gamma$ can be measured in a polarized microscope by compensation, thus allowing for a quantitative determination of the specimen birefringence $\Delta \mathrm{n}$.

For the theoretical case of perfect molecular alignment in a polymer sample, $\Delta \mathrm{n}=\mathrm{n}_{1}-\mathrm{n}_{2}$ where $\mathrm{n}_{1}$ and $\mathrm{n}_{2}$ are the refractive indices parallel and perperpendicular to the macromolecular chain axis, respectively. For the practical case of real oriented polymers, $\Delta \mathrm{n}$ represents an absolute measure of the degree of orientation if $n_{1}$ and $n_{2}$ are known. In the absence of this information, $\Delta \mathrm{n}$ can still be used as a quantitative measure to determine differences in the degree of orientation on a relative scale.

$\Delta \mathrm{n}$ values were measured for 19 individual LCP fibrils, taking the fibril diameter as representing the sample thickness, t, in Eq. (1). Fibril dimensions ranged from 0.3 to $12 \mu \mathrm{m}$ in diameter and from 6 to $280 \mu \mathrm{m}$ in length. As shown in Fig. 10, it was the shape factor of the fibrils (and, in particular, the length-to-diameter aspect ratio) which was found to be mainly responsible for the extent of birefringence and thus for the degree of molecular alignment. On the other hand, no significant trends were detected when the same $\Delta \mathrm{n}$ values were plotted directly as a function of individual fibril dimensions such as fibril diameter, fibril length or fibril volume (average fibril size).

\section{Discussion}

Skin-core effects are typical for injection-moulded products, and their influence on preferred macromolecular orientation is well known ${ }^{19,20}$. Standard orientation measurements by X-ray diffraction or infrared spectroscopy techniques which do not offer spatial resolution become more complicated in such cases, because a large number of samples have to be investigated in order to account for local variations and thus to specify the extent of core and skin regions with respect to orientation effects. At the beginning of this work, it was hoped that this shortcoming could be resolved by the technique of birefringence which, as a microscopic technique, would guarantee the spatial resolution of the light microscope (better than $1 \mu \mathrm{m}$ ), and promise at least a qualitative and comparative determination of the presence of preferred macromolecular orientation within this resolution limit. Certainly, this objective could be reached in the case of single-phase polymers ${ }^{21}$ but, as shown by the results presented in section 3.2, this may not be the case for multiphase materials.

According to Fig. 2, all three blend compositions exhibited skin regions with an approximate width of $1 \mathrm{~mm}$, an estimate based in this case only on the shape of the LCP particles. It is interesting to note that the same width of skin regions can be observed in Fig. 4, only this time based upon birefringence effects. However, the presence of birefringence also in transverse sections indicates that these birefringence effects are not related to molecular alignment only (orientation birefringence), but are also due to some cutting artifact based upon the particle morphology (form birefringence). Furthermore, as shown in Fig. 5, the elongated particles, embedded in the PPS matrix, did not generate any birefringence. Thus, even LCP fibrils would appear to be without preferred macromolecular alignment. However, the apparent lack of birefringence of the LCP particles in Fig. 5 is believed to be due to the complex nature of light transmission in a composite sample. In this case, the polarized light beam not only travels through oriented LCP particles but also through a non-oriented (spherulitic) matrix material above and below the particle, with possibly further complications due to the presence of the LCP/PPS interfaces.

At this stage of the research, the use of polarized light microscopy as a method for detection and quantitative measurement of macromolecular alignment in the PPS/LCP samples seemed to have lost its usefulness. On the other hand, the possibility of separating the LCP particles from the blends, discovered by chance, rendered new justification in favour of the birefringence technique. Before going into further detail, it should be recognized that the effect of particle aspect ratio on birefringence, as shown in Fig. 10, permits a direct correlation between two separate manifesta- 
tions of the skin-core effect: The first one based upon the geometrical aspects (fibrils or droplet particles) presented in Fig. 2, and the second one based upon molecular orientation which, as argumented above, is more difficult to obtain by standard X-ray diffraction or infrared spectroscopy techniques.

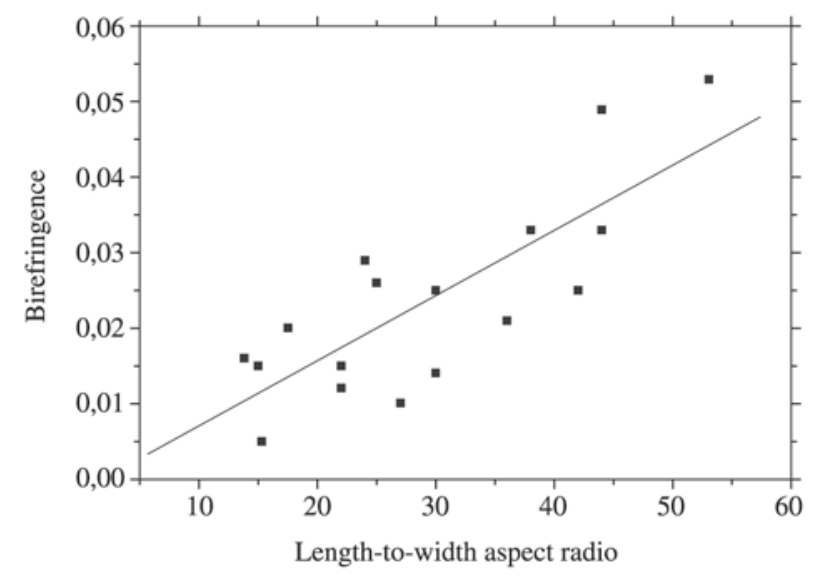

Figure 10. Birefringence of individual, elongated LCP particles as a function of their aspect ratio.

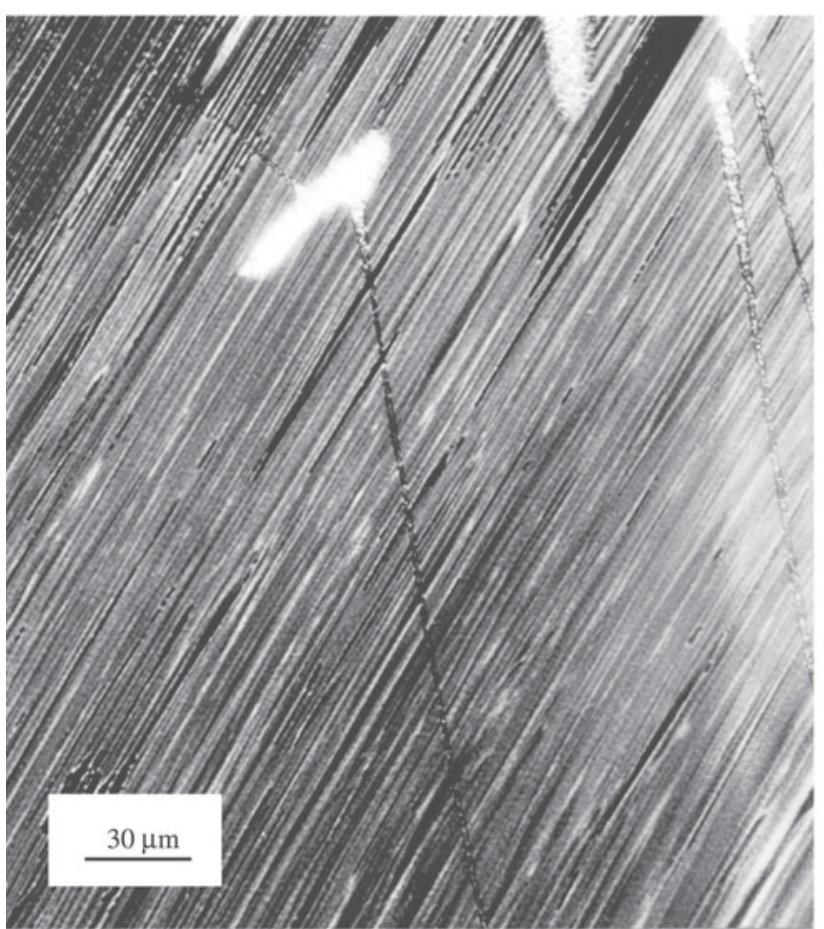

a)
As shown in Fig. 10, $\Delta$ n values for the LCP fibrils ranged from about 0.005 to 0.05 . In qualitative terms, it is certainly expected that the molecular alignment should be proportional to the length-to-width aspect ratio of the individual particles. In addition, however, and as shown in Fig. 10, there seems to be a quantitative linear relationship between the preferred orientation and the fibril morphology. It is this quantitative relationship which allows to equate in a rather general way particle morphology with molecular orientation effects, accentuating the significance of direct microscopy observations.

In order to put the linear relationship shown in Fig. 10 on absolute terms, the only thing which would be needed is the $\Delta \mathrm{n}$ value for the perfectly oriented LCP material. In the absence of such data, thin-film samples of the pure LCP phase were stretched (sheared) manually between glas slides in order to maximize their degree of macromolecular orientation. An example of such a sample is shown in Fig. 11. Birefringence measurements on this kind of material always exhibited $\Delta \mathrm{n}$ values which were higher than those encountered for the LCP fibrils elongated in blend samples by the injection moulding process. When evaluating quantitatively, $\Delta \mathrm{n}$ values for the pure LCP phase, produced by manual

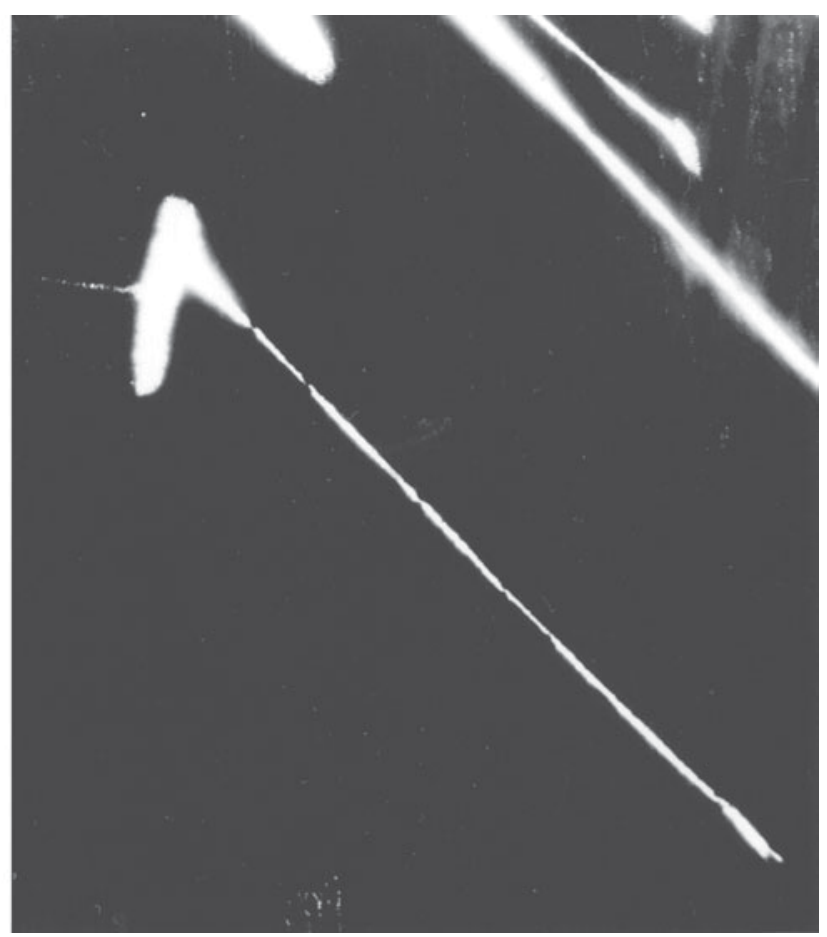

b)

Figure 11. Thin film of pure LCP phase showing birefringence after manual shearing. First shear left droplets and produced isolated fibrils only ("golf club" morphology). Second shear in a different direction produced continuous film sections. Under polarized light, continuous film section shows reinforcement in a), extinction in b). Orientation effect is reversed for "golf club" particles. Magnification 320×. 
shearing, varied between 0.037 and 0.095 . This range may be compared to some typical $\Delta \mathrm{n}$ values listed in the literature $^{22}$ and which indicate 0.062 for single PE macromolecules (accepted as the maximum theoretical value for perfect molecular alignment of PE fibers), 0.053 for cotton fibers, and 0.193 for highly stretched PET fibers. It is suggested that such numbers, if nothing else, are at least useful to render confidence to the quantitative procedure adopted in the present investigation.

Finally, when comparing the results of manual shearing of pure LCP $(\Delta \mathrm{n}$ values between 0.037 and 0.095$)$ with those obtained for LCP fibrils in 70/30 PPS/LCP blends ( $\Delta \mathrm{n}$ values between 0.005 and 0.05 ), it can be seen that the process of injection moulding, even in the skin regions where highly elongated LCP particles were formed, produced preferred macromolecular alignment on a partial scale only. It may therefore be suggested that the molecular alignment and, as a consequence, the directional properties of PPS/LCP blends could be improved by more appropriate processing conditions.

\section{Conclusions}

From the results obtained in the present investigation, the following conclusions may be drawn:

The determination of birefringence effects in polarized light microscopy represents a useful technique for the study of macromolecular orientation of LCP particles in PPS/LCP polymer blends.

For this purpose, LCP particles have to be separated from the PPS matrix. A simple separation technique which works has been shown to be the low-temperature cutting of thin PPS/LCP sample sections by microtomy.

After injection moulding, preferred molecular orientation was only found in skin regions where elongated LCP fibrils had formed.

The degree of preferred molecular orientation in the LCP phase was found to be proportional to the length-to-diameter aspect ratio of individual LCP fibrils.

In quantitative terms, the degree of molecular orientation of LCP fibrils separated from injection-moulded PPS/LCP blend samples was always less than the degree of molecular orientation which could be introduced into pure LCP samples by manual shearing.

\section{Acknowledgements}

The authors are grateful for financial support from
CAPES / DAAD (PROBRAL Proc.059/98), to G. Gabellini for providing the injection-moulded samples, and to R.E.S. Bretas for fruitful discussions.

\section{References}

1. Dutta, D.; Fruitwala, H.; Kohli, A.; Weiss, R.A. Polym. Engng. Sci., v. 30, p. 1005, 1990.

2. Bashir, Z.; Odell, J.A. J. Mater. Sci., v. 28, p. 1081, 1993.

3. Ballauf, M. Polym. Adv. Technol., v. 1, p. 109, 1990.

4. Schartel, B.; Wendorff, J.H. Polym. Engng. Sci., v. 39, p. $128,1999$.

5. Williams, G.E. Kunststoffe, v. 77, p. 1032, 1987.

6. Joseph, E.G.; Wilkes, G.L.; Baird, D.G. Polym. Engng. Sci., v. 25, p. 377, 1985.

7. Crevecoeur, G.; Groeninckx, G. Polym. Engng. Sci., v. 30. p. 532, 1990.

8. Gonzalez-Nunez, R.; Favis, B.D.; Lavallée, C. Polym. Engng. Sci., v. 33, p. 851, 1993.

9. Heino, M.T.; Hietaoja, P.T.; Vainio, T.P.; Seppälä, J.V. J. Appl. Polym. Sci., v. 51, p. 259, 1994.

10. Baird, D.G.; Ramanathan, R. Contemp. Topics Polym. Sci., v. 6, p. 73, 1989.

11. Weiss, R.A.; Wansoo, H.; Nicolais, L. Polym. Engng. Sci., v. 27, p. 684, 1987.

12. Hull, D. An Introduction to Composite Materials, Cambridge University Press, Cambridge, England, 1981.

13. Dreher, S.; Seifert, S.; Zachmann, H.G.; Moszner, N.; Mercoli, P.; Zanghellini, G. J. Appl. Polym. Sci., v. 67, p. $67,1998$.

14. Jasse, B.; Koenig, J.L. J. Macromol. Sci. Rev. Macromol. Chem., v. C17, p. 61, 1979.

15. Tadmor, Z.; Gogos, C.G. Principles of Polymer Processing, J. Wiley, New York, p. 607, 1980.

16. Blizard, K.G.; Baird, D.G. Polym. Engng. Sci., v. 27, p. $653,1987$.

17. James, S.G.; Donald, A.M.; MacDonald, W.A. Molec. Cryst. Liq. Cryst., v. 153, p. 491, 1987.

18. Haudin, J.M. in Optical Properties of Polymers, G.H. Meeten, ed., Elsevier Appl. Sci., London, p. 187, 1989.

19. Barres, O.; Friedrich, C.; Jasse, B.; Noël, C. Makromol. Chem., Macromol. Symp., v. 52, p. 161, 1991.

20. Bensaad, S.; Jasse, S.; Noël, C. Polymer, v. 34, p.1602, 1993.

21. Hobbs, S.Y. Polymer Microscopy, General Electric Report $\mathrm{N}^{\circ} 80 \mathrm{CRDO} 25$, p. 10, 1980.

22. Brandrup, J.k.; Immergut, E.H.; Polymer Handbook, eds., J. Wiley \& Sons, New York, 1975. 